\title{
Modeling and Simulation Enabled UAV Electrical Power System
}

\author{
S. Fletcher, P. Norman, P. Rakhra, S. Galloway, G. Burt \\ University of Strathclyde
}

Copyright (C) 2011 SAE International

\begin{abstract}
With the diversity of mission capability and the associated requirement for more advanced technologies, designing modern unmanned aerial vehicle (UAV) systems is an especially challenging task. In particular, the increasing reliance on the electrical power system for delivering key aircraft functions, both electrical and mechanical, requires that a systems-approach be employed in their development. A key factor in this process is the use of modeling and simulation to inform upon critical design choices made. However, effective systems-level simulation of complex UAV power systems presents many challenges, which must be addressed to maximize the value of such methods. This paper presents the initial stages of a power system design process for a medium altitude long endurance (MALE) UAV focusing particularly on the development of three full candidate architecture models and associated technologies. The unique challenges faced in developing such a suite of models and their ultimate role in the design process is explored, with case studies presented to reinforce key points. The role of the developed models in supporting the design process is then discussed.
\end{abstract}

\section{INTRODUCTION}

Unmanned Aerial Vehicles (UAVs) are being developed for use in both civil and defense applications to capitalize on the potential of autonomous performance. Modern UAV system designs are likely to employ the More-Electric Aircraft (MEA) concept in order to enhance efficiency and improve the availability and maintainability of aircraft systems [1].

These modern UAV systems are likely to be far less dependent on legacy equipment and as such there are fewer constraints on the electrical system design and architecture [1,2]. The greater flexibility offered creates the opportunity for more integrated electrical system design and greater system optimization. However by relaxing system design constraints, a number of new variables are introduced (e.g. voltage level, system frequency, energy storage usage, load type etc) which presents additional challenges in achieving the optimal design. Given the complexity of this task, accurate network modeling and simulation is an essential element in de-risking the design process for new systems, by enabling the trialing of new technologies and operational concepts. However, the challenges associated with this process for UAV power systems are not well documented in the research literature.

This paper presents the models developed for three candidate twin generator power system architectures (supplying a common set of loads) for a medium altitude long endurance (MALE) UAV application. These architectures form the basis of an investigation into optimal power distribution method and network design.

The paper addresses a number of key modeling issues faced in the development of high fidelity electrical system models and discusses measures taken to overcome these. This includes capturing both small and large time constants in the simulation, such as interactions between electrical components and between the electrical power system and aircraft propulsion system respectively, in a computationally efficient and yet accurate manner. Furthermore, achieving model validation of system-level behavior of a conceptstage power system, maintaining modeling consistency between candidate architectures (for fair comparative simulation studies) and implementing a flexible modeling framework to facilitate the simulation based investigation of a wide range of scenarios.

With the modeling capability established, methods of analyzing and evaluating the performance of a UAV electrical system architecture against a number of defined criteria are investigated. In particular, the paper describes the utilization of these models to assess the applicability of an example backup battery before illustrating the response of the network to a variable load profile, and

Page 1 of 12 
simulation results are shown to demonstrate this capability. Finally conclusions are drawn regarding the use of the developed models in supporting this particular design process and the future work required to maximize their effectiveness.

\section{DESIGN CHANGES WITH ADVANCEMENTS IN TECHNOLOGY}

Development of power system component technologies, such as power electronics and electric drives, is enabling improvements in aircraft electrical systems performance and availability [3,4]. The potential for these system improvements is the driver behind much recent research into the design of novel aircraft electrical system architectures.

This is perhaps most evident from the progressive shift from $115 \mathrm{~V}$ three-phase AC toward utilizing $270 \mathrm{~V}$ DC for primary power distribution in modern UAV electrical network designs to capitalize on the many key advantages DC offers [5]. By utilizing DC power distribution, it is often possible to reduce the number of power converters employed in a specific network design, especially where frequency wild generation $[1,6]$ or novel loads, with unique voltage and frequency requirements, are employed. Elimination of such conversion stages may significantly reduce converter operating losses and the weight/volume of the network. Furthermore, DC distribution readily permits the paralleling of multiple generators onto a single bus, a practice which potentially enables increased whole system efficiency during part load operation, increased design flexibility and redundancy, and reduced through-life costs [7]. This can be particularly beneficial when paralleling Low Pressure (LP) and High Pressure (HP) shaft mounted generators [4], as the operability and performance of the engine can be actively improved [6] by operating the generators at a wider speed range and transferring power between turbine shafts.

However, the lack of lightweight and effective solutions for electrical fault protection of higher voltage DC systems represents a significant barrier to more widespread adoption of DC within aircraft systems $[8,9]$. These issues include the potential for high magnitude, rapidly occurring fault currents $[10,11]$, which result from the discharge of filter capacitors through limited impedance interconnecting cables. The design of effective protection for DC systems is an area of ongoing research. The overriding challenge in terms of network design is to ensure that the significant benefits attained through the utilization of DC distribution are not negated by the requirement to accommodate additional protection equipment or network redundancy.

Energy storage within aircraft systems are traditionally employed to supply backup power for flight critical loads in the event of primary generation failure, and usually take the form of battery systems. However advanced energy storage devices can play a number of additional roles within electrical power distribution systems. To enable energy storage to become more effective, a combination of both battery and supercapacitor systems are being considered for use on aircraft electrical systems [12, 13]. Each of these storage mediums have distinct dynamic performance [14]. The power density and charge/discharge life cycle of supercapacitors can be considerably greater than that of battery systems, whereas the energy density of batteries can be up to two orders of magnitude than supercapacitors [14]. Thus, employment of both storage mediums can be complementary, providing the capability for both fast transient mitigation (which can benefit both engine and electrical system operation) as well as longer term backup supply, potentially extending the range of possible applications within the aircraft electrical system.

\section{UAV NETWORK ARCHITECTURES}

This section presents three modeled UAV power system architectures; a variable frequency AC (VFAC) baseline network, and functionally consistent hybrid AC/DC and full DC variants. These architectures have been selected to cover a range of likely distribution options, capturing both traditional and novel architectures in order to provide a basis for comparison. This approach does not necessarily maximize the potential benefits of each distribution means, however it is anticipated the analysis of the three networks will inform upon the optimal network architecture for future studies.

The network models have been developed as behavioral level models [15], capturing converter switching effects, and load and fault transients. Modeling work has been conducted within the Simulink and SimPowerSystems blocksets of Matlab [16].

\section{VFAC ARCHITECTURE}

The modeled VFAC architecture is illustrated in Figure 1. 


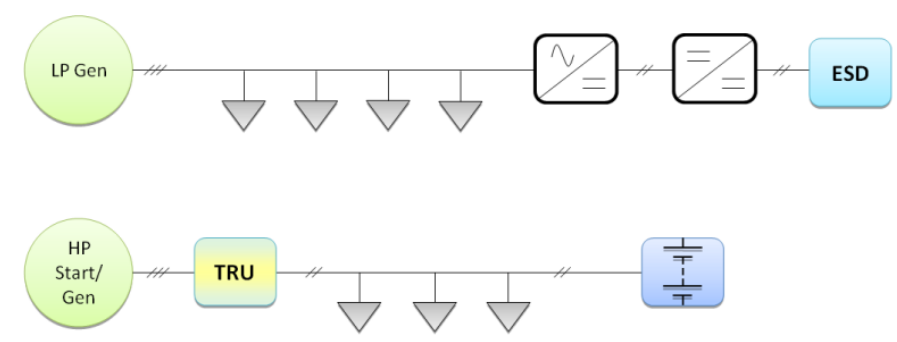

Figure 1: VFAC UAV power system schematic

This network contains two 3-phase, variable-frequency, synchronous generators, which are driven from the low pressure (LP) and high pressure (HP) shafts of an aircraft engine respectively. Generator Control Units (GCUs) are also modeled to regulate the voltages at the primary busses to $115 \mathrm{Vrms}$ (line-line). A representative aircraft engine model is employed to provide a rotational speed profile input for the generator and can be adjusted according to the mission status (i.e. take-off, landing and cruise profiles). The engine model is currently represented as a speed-stiff system, whereby changes in electrical loading on the generator do not induce changes in rotor speed. A supercapacitor energy storage device (ESD) has been modeled based on that presented in [17] and is interfaced to the network via a controlled bi-directional DC-AC converter. The applied converter control system is based on a power-balance strategy developed in [17], incorporating tracking of load changes on the network and whilst maintain the charge on the supercapacitor. A Transformer Rectifier Unit (TRU) converts the 115V VFAC supply to 28VDC for distribution to the DC bus. The DC bus also contains an additional battery storage system model to provide an emergency backup supply to the loads on this bus.

\section{HYBRID AC/DC ARCHITECTURE}

Figure 2 shows the schematic of the modeled hybrid AC/DC architecture.

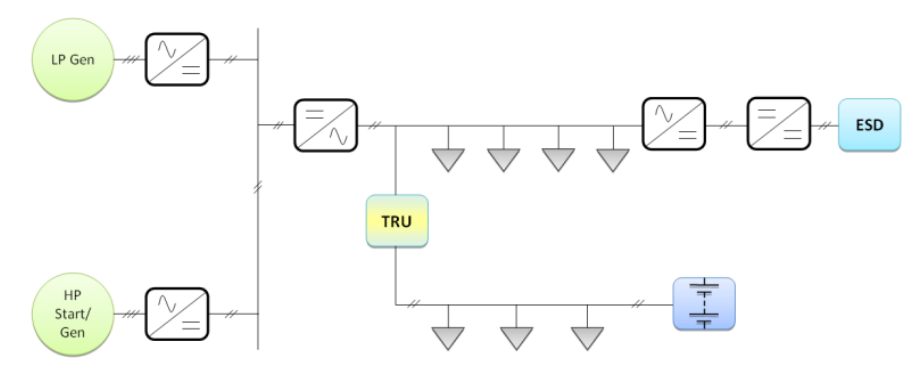

Figure 2: Hybrid DC/AC UAV power system schematic

The hybrid AC/DC network architecture captures key benefits of a DC busbar whilst retaining the more common 115VAC distribution to loads for protection compatibility. In this network, the output of the two generators is now rectified (using voltage source converters and phase angle control [18]), enabling easier paralleling of and power sharing between generators. Tuned droop profiles were employed within the converter interface controllers to ensure optimal power sharing between HP and LP driven generators, as illustrated in Figure 3 below. At present, equal maximum and minimum voltages have been utilized for improved voltage regulation of the rectifier outputs. However as [19] reports, greater machine efficiency can be achieved with a different voltage range on either generator control. There is also potential for the power split between the two generators to be regulated to optimize engine operability throughout different phases of the aircraft flight cycle [20]. Greater knowledge of both the engine's and two machines' efficiency against load characteristics may allow these benefits to be maximized in future. The rectified generator output also provides opportunity for wider generator speed range operation of the aircraft engine and the introduction of power dense generator technologies. 


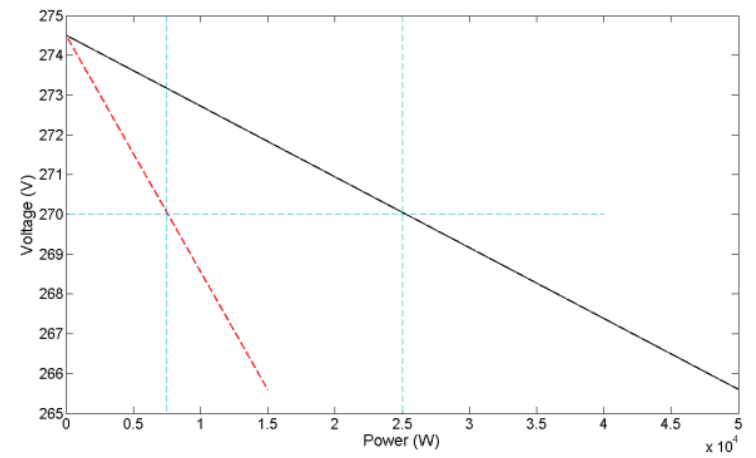

Figure 3: Voltage droop profile for the LP (solid line) and HP (dotted line) rectifier control systems

Other important features for this network include the use of a single inverter interface to supply 115Vac loads with a TRU connected to this supplying the 28VDC bus.

\section{ARCHITECTURE}

The schematic in Figure 4 represents the third UAV electrical system architecture considered within this paper, where power is now distributed through a 270VDC ring network. Within this network, the DC ring provides the opportunity to reduce the number of power conversion stages between generation, energy storage and loads, potentially increasing overall efficiency. The ring architecture of the 270VDC network section also utilizes parallel supply paths to loads, providing increased security of supply.

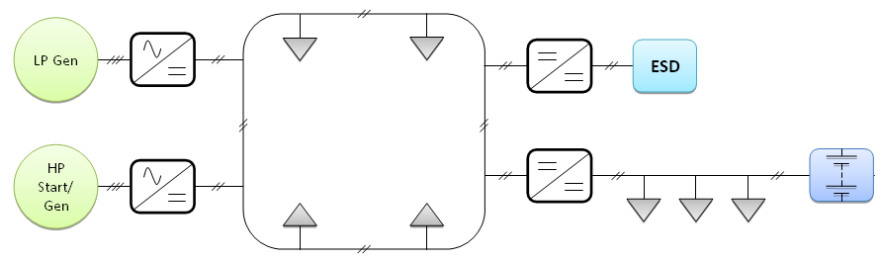

Figure 4: DC UAV power system schematic

\section{MODELLING CHALLENGES}

The high-fidelity UAV power system models which have been developed for the networks presented in the previous section require significant computational resources in order to carry out meaningful technical and temporal simulations. To address this issue, several measures have been taken, incorporating the modeling constraints that exist in this study. These aspects are discussed in the following sections.

Given the wide range of studies anticipated during the network comparison activities, the UAV power system models have been developed at a behavioral level [15]. In this manner, power electronic converter models operating with ideal switches and detailed $6^{\text {th }}$ order machine models have been employed. Grouped loads (both fixed and dynamic) and a speed-stiff aircraft engine representation are also used, where the studies permit, in order to reduce overall computational requirements [21]. In line with guidance given in [22], a fixed step solver is employed to enable the most computationally efficient simulation of the multi-converter networks considered. Provision for multi-rate simulation has also been made when more detailed representations of the aircraft engine model are employed to avoid the unnecessary over-computation of this component when incorporated within the wider electrical network models.

It is noted however, that for many of the anticipated simulations, the behavioral approach provides more simulation detail than is actually required. In these cases, the functional approach, where devices with high frequency characteristics (such as power electronic converters) are represented in a time averaged fashion in order to reduce the overall simulation computational requirement and permit the use of larger solver step sizes is preferable. Indeed, this approach can be readily implement within the DC ring network model whose power electronic converter topologies are all of the fully controlled type (and hence amenable to average-value representation). 
However, in the baseline VFAC and hybrid AC/DC networks, the uncontrolled rectifiers within the TRUs are substantially more challenging to represent at the functional level. The co-dependence between the converter's operating state and the surrounding network state requires unique approaches to be employed before the time averaged behavior of the converter can be modeled. Whilst there has been considerable research in this field [23], and more recently with specific systems-level considerations [24-26], there is no existing technique which is both readily compatible with the network models developed and offers full multimode operation that could be employed. As such, the lowest acceptable level of fidelity for the VFAC and hybrid AC/DC network models is the behavioral level.

This conclusion regarding the VFAC and hybrid AC/DC models has interesting consequences for the DC ring network architecture. In future comparison studies, would it be feasible to compare the functional level outputs from the DC ring network model with the behavioral level output of the hybrid AC/DC and VFAC network models and draw meaningful and valid conclusions? The nature of each study considered may also impact on the outcome of this question. Given this uncertainty, behavioral models of all networks (for the immediate term at least) were employed to attain consistency in the comparison of simulation results. In future, with appropriate testing, it may be deemed acceptable to do comparisons of behavioral level simulation results with functional level results. Additionally, as indicated by the authors in [22], a hybrid behavioral/functional representation of the hybrid AC/DC and VFAC network models may be an acceptable compromise. In this approach, the diode rectifiers are represented at behavioral level whilst all other converters are represented at the functional level.

\section{VALIDATION CHALLENGES}

The testing and evaluation of the validity of the UAV network models is a key phase of their development. Law [27] describes the validation of a model as a process to determine whether it is an acceptably accurate representation of the system within the context of a given study. The availability of hardware test rigs and data, third party models and simulation results, and expert knowledge on the technologies present within the three UAV network models identified in this paper is such that the validation of individual subsystem models employed within these has been fairly readily achieved.

Whilst the use of validated subsystem models might be expected to be sufficient in guaranteeing an accurate whole system model, the authors' experience in previous projects has shown that the complex nature of the interactions between subsystems, and the potential for incompatible validation assumptions can produce unexpected emergent behavior. As such, additional system-level validation is also beneficial. In principle, the validation methods applied to the subsystems are equally applicable to the validation of the integrated model. In practice, however, a number of difficulties arise.

The use of predictive validation (comparison of simulation results to hardware testing [27]) would provide the greatest degree of confidence in the model operation. Construction of a dedicated hardware test rig for each of the three network models, whilst possible, is very costly and negates many of the economic benefits of using simulation to de-risk technology at the design stage. The range of equipment and configuration options which could be investigated is also limited in this approach. Utilizing test/commissioning data from existing similar UAV/aircraft power systems or demonstrators provides a possible alternative, although the limited availability of these and the potentially confidential nature of any data captured substantially reduces the likelihood of this approach providing a meaningful option. Published data and results from the Intelligent Electrical Power Network Evaluation Facility (IEPNEF) testing at the University of Manchester [17] and other similar projects, for example, may provide the opportunity to validate some elements of the hybrid AC/DC and DC ring network models' behavior. This approach however, is not expected to provide any significant degree of coverage to validate the behavior of the full system model.

The comparison of model behavior to that of previously validated or independently developed models [27] offers slightly more potential than the previous approaches considered. The availability of simulation results from other aircraft electrical systems modeling projects is greater than that for hardware test rigs and covers a greater range of operating modes. However, the likelihood of obtaining an independently developed model or suite of models purely for the purposes of validating the three UAV network models developed is rare, and as such, this approach cannot be considered as a primary tool in achieving good validation coverage.

Finally, face validation, in which opinions are sought from one or more experts regarding the acceptability of the model's construction or behavior provides a useful, if subjective, approach where many aspects of a model's behavior can be qualitatively validated. It is particularly useful in addressing the 'gaps' left using other validation approaches. Use of formal approaches to capture this expert knowledge [28] also extends range within which this validation approach can be applied.

In light of the limitations discussed above, systems-level face validation (supported by predictive and model comparison based validation where possible) combined with thorough validation of individual subsystems and components is perhaps the best practically

Page 5 of 12 
achievable solution. To this end, it has been the approach employed in the development of the three UAV networks presented in previous sections.

\section{DESIGN APPROACH}

As discussed in previous sections, the initial design approach being taken within this project is to identify three electrical distribution architectures and then comparatively evaluate these (supported my modeling and simulation) against a set of design attributes. The desired outcome of this evaluation is to identify the preferred distribution option for a given application. Secondary goals also include the identification of key technologies and technological constraints through this process. Karimi [29] identifies a number of attributes upon which one can evaluate an aircraft electrical system design. These attributes include:

- $\quad$ Power system efficiency

- Weight

- Volume

- Total Cost

- Safety

- Thermal Efficiency

- Reliability

- Maintainability

- Functionality

- Cost Effective Rapid Technological Insertion

- Green Systems

A common approach taken to evaluating (and/or optimizing) the design of any system is to use a weighted sum [30]. Within this approach, each of the design attributes is given a weighting based on their relative importance to the application in consideration. Each particular network configuration is then given a rating against each design attribute using an appropriate numerical scale, which is multiplied by its respective weighting. The sum of these individually weighted ratings gives an overall score for the network, which indicates how well it matches a specific design objective. This score also allows comparison with other, similarly rated, networks. However, to enable such a network assessment to be carried out, a number of questions must first be addressed. These include, what is the relative importance of the design attributes? What are the criteria for rating a network against each of these attributes? How do these change will these factors change with aircraft size or application area? Each of these questions will be relevant for any quantitative or more detailed optimization method [31] and therefore achieving a design solution based on this comparison approach is a significant research challenge.

Whilst many of these design factors can be judged in a subjective and qualitative manner, providing a quantitative measure of network attributes enables more informed decision making to be made in the design process. Accurate network modeling can be a key element for supplying much of this quantitative information. For example, models can readily be used to demonstrate aspects of a network's safety through the simulation of fault conditions and testing of protection system operation. This is also the case for other attributes such as functionality or system efficiency. The following sections will illustrate the operation of the developed system models to demonstrate their capability to contribute to this design process.

\section{ILLUSTRATION OF MODEL OPERATION}

This section will illustrate the operation of some component and network models for three UAV power system architectures described in the previous sections. Due to the space constraints of this paper, the section will focus on the illustration of one individual component model and one integrated system model. 


\section{BATTERY SYSTEM}

A battery system is connected to each of the networks at 28VDC and its primary role is to supply the loads on this bus in the event of a loss of supply from main generation. Nickel cadmium has long been utilized as a battery technology for aircraft systems [32, 33], and as such has been adopted initially for the three network architectures. Example voltage and capacity parameters are contained within [34], which can be used to populate a standard battery model [35]. Parameters for this model are presented in table 1 and to demonstrate the performance of the battery model, figure 5 illustrates the discharge curves under varying conditions.

Table 1: Battery model parameters

\begin{tabular}{|l|l|}
\hline Battery type & Nickel Cadmium \\
\hline Nominal voltage & $24 \mathrm{~V}$ \\
\hline Rated capacity & $43 \mathrm{Ah}$ \\
\hline
\end{tabular}
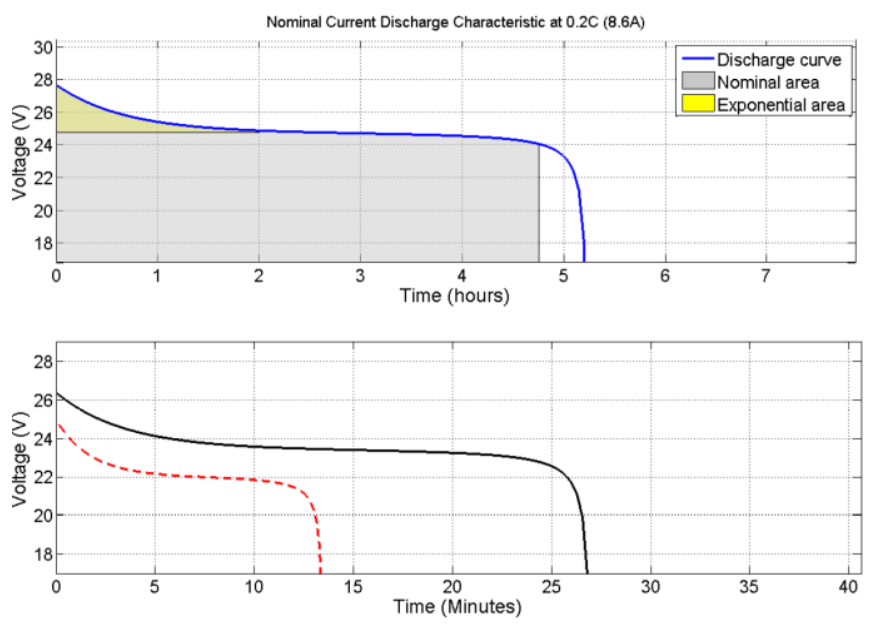

Figure 5: Nickel cadmium battery discharge curves for voltage against time

Figure 5 illustrates the discharge plots of the battery model by plotting its terminal voltage against time for three discharge currents. The upper plot shows a discharge curve for a nominal current output of 8.6A. This plot shows a fully charged voltage of $28 \mathrm{~V}$, which exponentially decays to the nominal level of $24 \mathrm{~V}$. This full charge voltage and exponential decay zone are dependent on the battery type selected for the model. The bottom plot in figure 5 compares the discharge curves for much faster discharge rates of 100A (solid line) and 200A (dotted line) respectively. These higher discharge rates are representative of the required current output to support critical loads under loss of supply conditions. For the 200A discharge curve, figure 5 shows that a load of around $5 \mathrm{~kW}$ on the $28 \mathrm{Vdc}$ bus can be supplied for around 10 minutes, albeit at a lower than normal voltage level, before the battery voltage begins to decay rapidly. The figure demonstrates the capability of the model to readily assess the performance of different battery systems for a range of current discharge conditions and hence aid in the selection of appropriate battery types and capacities for the candidate network architectures. It also permits future studies into the optimal energy storage arrangement (potentially comprising of both batteries and supercapacitors) for various applications.

\section{GENERIC DYNAMIC LOAD MODEL}

In order to be able to represent a variety of novel loads within the three network architecture models, a generic dynamic load model has been developed. This model has been designed as a 'black box' system, connectable to any three-phase ac supply (of fixed or varying frequency), and with inputs of magnitude and phase for each of the three phase currents. In this manner, load magnitude, power factor and imbalance can be controlled dynamically, while the integration of the load model within the network model is seamless.

The controllable load model consists of two main parts. The first component is a Simulink-based synchronization and control element. The functionality of this system is illustrated in figure 6 below. 


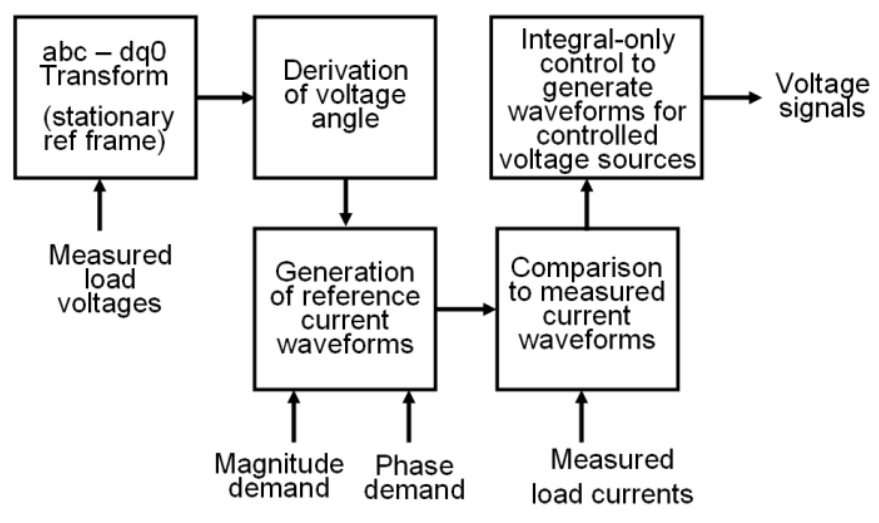

Figure 6: Load model control element

The control element operates by measuring the three phase voltages at the terminals of the controllable load and transforming these into a stationary dq0 reference frame. The dq voltages are then manipulated to extract the angle of the phase ' $\mathrm{a}$ ' voltage. Note that this approach assumes a balanced three phase voltage across the load terminals to enable accurate extraction of voltage angle. However, under testing with an imbalanced supply, the controllable load has still achieved acceptable levels of load current regulation although some distortion is present in the waveforms.

In conjunction with the voltage reference angle, load current reference waveforms can be constructed using magnitude and phase angle demands for each load phase. These waveforms are compared to measured load currents to produce current error signals, which are subsequently fed into high-gain integral-only controllers to generate control signals for the three controlled voltage sources in the circuit element of the controllable load. Note that an integral-only control is employed to provide a natural break in the algebraic loops that would otherwise exist in this model if a proportional or derivative gain was employed.

The circuit element of the controllable load employs three controlled voltage sources in series with three fixed resistances. This configuration was chosen over a controlled current source configuration as it was found to provide greater numerical stability during highly transient conditions. The magnitude of the series resistors is chosen to provide the optimum balance between the dynamic response of the load and its numerical stability under transient conditions.

\section{Simulated load profile}

To illustrate the operation of the controllable load model, a representative electromechanical actuator (EMA) startup load profile is simulated [36] with the controllable load model. Whilst the load magnitude is not necessarily representative of a UAV actuator, it does provide a useful illustration of the capability of the dynamic load model to represent highly transient loading conditions as well as showing the subsequent network response to these. For this particular case study, the load model is incorporated into the baseline VFAC network and is supplied by the LP spool driven synchronous generator. The network also features $8 \mathrm{~kW}$ of resistive loading on the $230 \mathrm{Vac}$ feeder but does not include the ESD system. The $28 \mathrm{Vdc}$ element of the network is also not represented for the purposes of this case study.

Figures 7 to 10 show rms traces of the simulated EMA load current profile (as measured at the terminals of the load model), a close up of load current waveforms compared to the current (peak) demand input to the load model, the total generator load current and generator terminal voltage (line-line). 


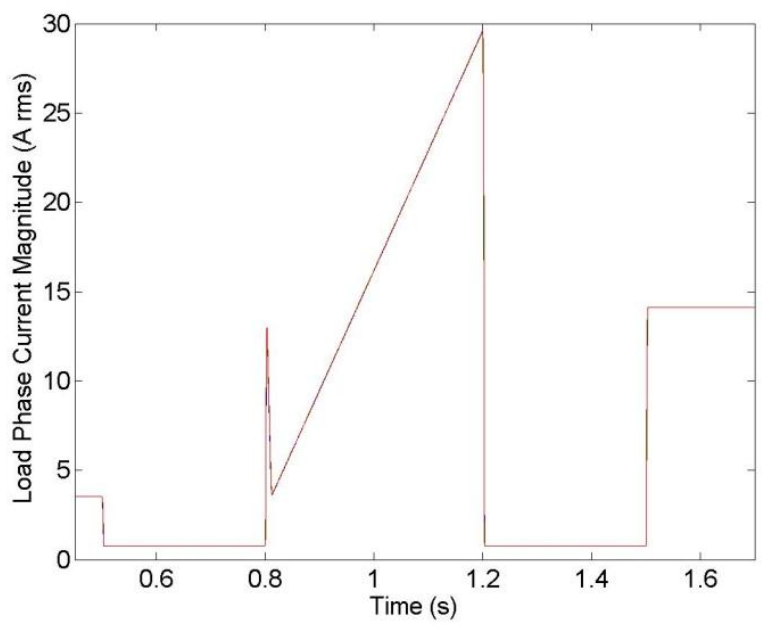

Figure 7. Simulated load current magnitude (A rms)

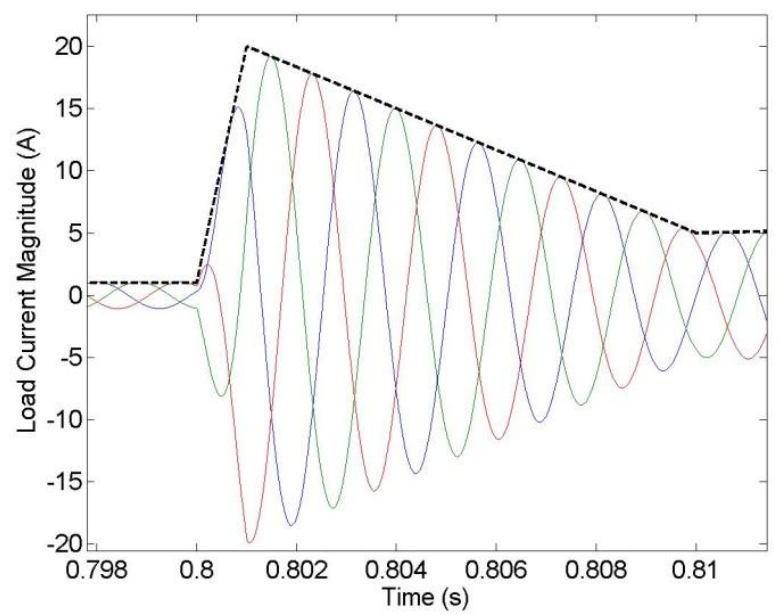

Figure 8. Simulated load current waveforms compared to load model demand profile (A rms)

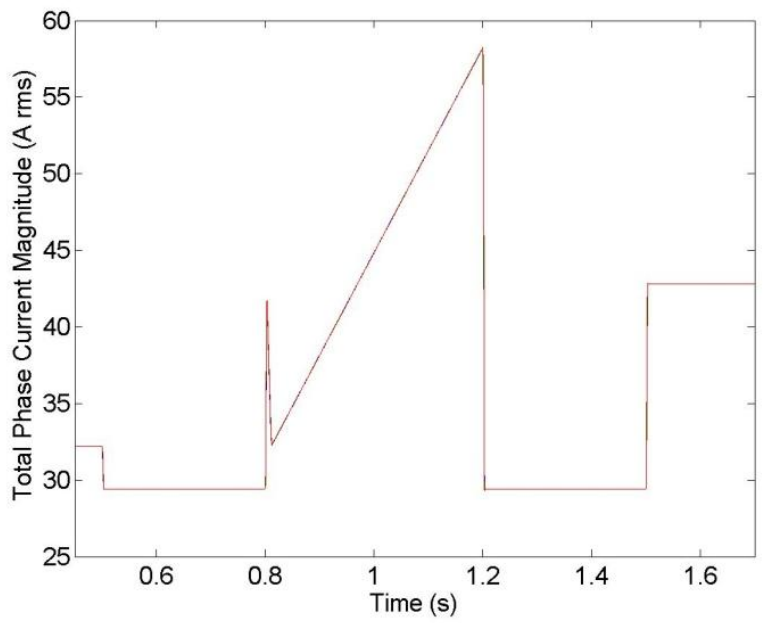

Figure 9. Simulated total generator current magnitude (A rms)

Page 9 of 12 


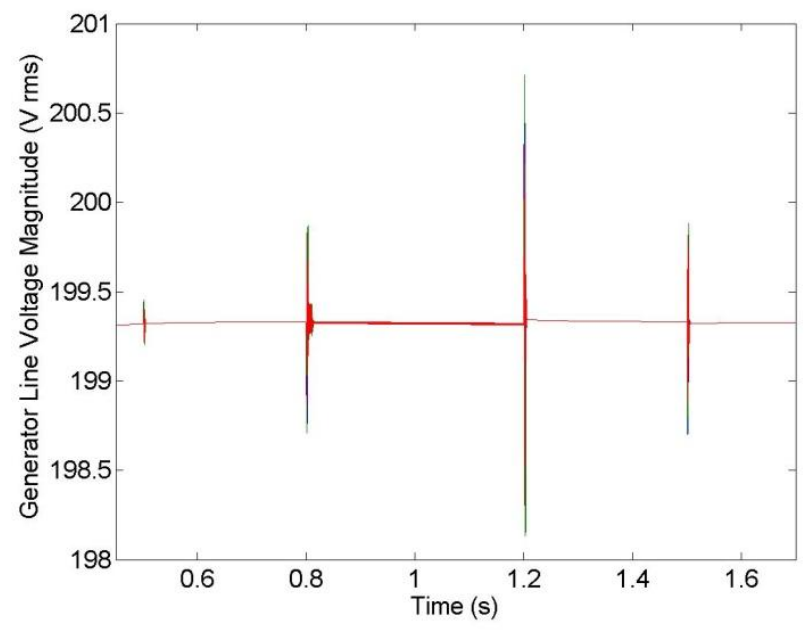

Figure 10. Simulated generator terminal voltage (V rms)

The results presented in figures 7 to 10 above illustrate the excellent reference tracking of the generic dynamic load model and the impact of highly transient loading conditions on the network operation. The GCU model employed also achieves good regulation of the network voltage during these conditions, although this could be further improved through the utilization of the supercapacitor ESD to improve network power quality [37]. This case study also provides some illustration of the capability and flexibility of the developed network models and components to be used in support UAV network design. In particular, as the generic dynamic load model enables the simulation of a variety of novel load profiles, the capability of the network to support these loads can be readily assessed, informing analysis on network functionality and technology insertion.

\section{CONCLUSIONS}

This paper has presented three candidate electrical power system architecture models developed in support of a MALE UAV power system design project. The modeling challenges faced in this process and the constraints on applicable solution methods were discussed before the results of two case studies were presented to illustrate the developed capability. Clear challenges lie ahead in terms of further refining the modeling capability and verifying the acceptability of comparing simulation results produced by models of different fidelity. Establishing the specific roles and considering novel uses of modeling and simulation in the definition of attribute scoring will also be of significant interest in the next phase of this UAV power system design project.

\section{REFERENCES}

1. Rosero, J., Ortega J., Aldabas, E., and Romeral, L., "Moving towards a more electric aircraft," Aerospace and Electronic Systems Magazine, IEEE, vol. 22, no. 3, pp. 3 -9, March 2007

2. Sinnet, M., "787 No-Bleed systems: saving fuel and enhancing operational efficiencies," in Boeing Commercial Aeromagazine, Quarter 4, 2007, pp. 6- 11.

3. Weimer, J. A., "Electrical power technology for the more electric aircraft," in Digital Avionics Systems Conference, 1993. 12th DASC., AIAA/IEEE, 1993, pp. 445-450.

4. Mitcham, A. J. and Cullen J. J. A., "Permanent magnet generator options for the More Electric Aircraft," in Power Electronics, Machines and Drives, 2002. International Conference on (Conf. Publ. No. 487), 2002, pp. 241-245.

5. Fletcher, S., Norman, P. J., Galloway, S. J., and Burt, G. M., "Evaluation of overvoltage protection requirements for a DC UAV electrical network," presented at SAE Power Systems Conference, November 2008, paper no. 2008-01-2900.

6. Chang, J., Wang A., "New VF-power system architecture and evaluation for future aircraft," IEEE Transactions on Aerospace and Electronic Systems, vol.42, no.2, pp. 527- 539, April 2006

7. Provost, M. J., "The More Electric Aero-engine: a general overview from an engine manufacturer," in Power Electronics, Machines and Drives, 2002. International Conference on (Conf. Publ. No. 487), 2002, pp. 246-251.

8. Long, S. A. and Trainer, D. R., "Ultra-compact intelligent electrical networks," in 1st SEAS DTC Technical Conference, 2006.

9. Norman, P. J., Galloway, S. J., Burt, G. M., Trainer, D. R., and Hirst, M., "Transient analysis of the more-electric engine electrical power distribution network," in Power Electronics, Machines and Drives, 2008. PEMD 2008. 4th IET Conference on, April 2008, pp. 681 -685.

Page 10 of 12 
10. Cuzner, R. and Venkataramanan, G., "The status of DC micro-grid protection," in Industry Applications Society Annual Meeting, 2008. IAS '08. IEEE, Oct. 2008, pp. 1-8.

11. Baran, M. E. and Mahajan, N. R., "Overcurrent protection on voltage source-converter-based multiterminal dc distribution systems," Power Delivery, IEEE Transactions on, vol. 22, no. 1, pp. 406 -412, Jan. 2007.

12. He Zhang, Mollet, F., Saudemont, C., Robyns, B., "Experimental Validation of Energy Storage System Management Strategies for a Local DC Distribution System of More Electric Aircraft," Industrial Electronics, IEEE Transactions on , vol.57, no.12, pp.3905-3916, Dec. 2010 doi: 10.1109/TIE.2010.2046575

13. Khaligh, A., Zhihao, Li, "Battery, Ultracapacitor, Fuel Cell, and Hybrid Energy Storage Systems for Electric, Hybrid Electric, Fuel Cell, and Plug-In Hybrid Electric Vehicles: State of the Art" IEEE Transactions on Vehicular Technology, Volume: 59, Issue:6, pp.2806 - 2814, July 2010

14. Musolino, V. and Tironi, E., "A comparison of supercapacitor and high-power lithium batteries," in Electrical Systems for Aircraft, Railway and Ship Propulsion (ESARS), 2010, 2010, pp. 1-6.

15. Wu, T., Bozhko, S.V., Asher, G.M., Thomas, D.W., "Fast functional modelling of the aircraft power system including line fault scenarios," Power Electronics, Machines and Drives (PEMD 2010), 5th IET International Conference on, no., pp.1-7, 19-21 April 2010 doi: 10.1049/cp.2010.0049

16. The Mathworks, "SimPowerSystems,", published online at http://www.mathworks.com/products/simpower, assessed 19/5/11

17. Todd, R., Wu, D., dos Santos Girio, J.A., Poucand, M., Forsyth, A.J., , "Supercapacitor-based energy management for future aircraft systems," Applied Power Electronics Conference and Exposition (APEC), 2010 Twenty-Fifth Annual IEEE , vol., no., pp.1306-1312, 21-25 Feb. 2010

18. Fernando, W.U.N., Barnes, M., Marjanovic, O., "Direct drive permanent magnet generator fed AC-DC active rectification and control for more-electric aircraft engines," Electric Power Applications, IET, vol.5, no.1, pp.14-27, January 2011

19. Adefajo, O.R., Barnes, M., Smith, A.C., Long, S.A., Trainer, D.R., Abdel-Hafez, A., Forsyth, A., Chivite-Zabalza, J., Cross, A., Todd, R., "Voltage control on an uninhabited autonomous vehicle electrical distribution system," Power Electronics, Machines and Drives, 2008. PEMD 2008. 4th IET Conference on , vol., no., pp.676-680, 2-4 April 2008

20. Hirst, M.; McLoughlin, A.; Norman, P.J.; Galloway, S.J.; , "Demonstrating the more electric engine: a step towards the power optimised aircraft," Electric Power Applications, IET, vol.5, no.1, pp.3-13, January 2011

21. Gole, A. M., Keri, A., Nwankpa, C., Gunther, E. W., Dommel, H. W., Hassan, I., Marti, J. R., Martinez, J. A., Fehrle, K. G., Tang, L., McGranaghan, M. F., Nayak, O. B., Ribeiro, P. F., Iravani, R., Lasseter, "Guidelines for modeling power electronics in electric power engineering applications," IEEE Trans. on Power Delivery, Vol. 12, No. 1, pp. 505 - 514, January 1997.

22. Jin, H., "Behaviour-mode simulation of power electronic circuits," IEEE Trans. on Power Electronics, Vol. 12, no. 3, pp. 443 452, May 1997.

23. Chinniforoosh, S. et al, IEEE Task Force on Dynamic Average Modelling, "Definitions and applications of dynamic average models for analysis of power systems," IEEE Transactions on Power Delivery, Oct. 2010, Vol. 25, No. 4, October 2010, pp. $2655-2669$.

24. Cross, A., Baghramian, A., Forsyth, A., "Approximate, average, dynamic models of uncontrolled rectifiers for aircraft applications," IET Power Electronics, Vol. 2, Issue 4, 2009, pp. 398 - 409.

25. Areerak, K. N., Bozhko, S. V., Asher, G. M., Thomas, D. W. P., "Stability analysis and modelling of ac-dc system with mixed load using dq-transformation method," ISIE '08, pp. 19 - 24, 2008.

26. Jatskevich, J., Pekarek, S. D., Davoudi, A., "Fast procedure for constructing an accurate dynamic average-value model of synchronous machine-rectifier systems," IEEE Transactions on Energy Conversion, Vol. 21, No. 2, June 2006, pp. 435 - 441.

27. Law, A.M., 2005. How to build valid and credible simulation models, Proceedings of the 2005 Winter Simulation Conference, December 4-7, Orlando, USA

28. CommonKADS, "Engineering and Managing knowledge", http://www.commonkads.uva.nl/, June 2011

29. Karimi, J., Boeing Technical Fellow, "Future aircraft power systems - integration challenges," available at: http://www.ece.cmu.edu/ electriconf/2008/PDFs/Karimi.pdf, accessed 19.5.2011

30. Scholz, D., Aircraft Systems - Reliability, Mass, Power and Costs, European Workshop on Aircraft Design Education 2002, Available online at http://www.fzt.haw-hamburg.de/pers/Scholz/ewade/2002/Scholz.pdf accessed 27/5/11

31. Marler, R. T. and Arora, J. S., "Survey of multi-objective optimization methods for engineering", in Structural and Multidisciplinary Optimization, Volume 26, Number 6, pp. 369-395, DOI: 10.1007/s00158-003-0368-6

32. Sobel, I. J.; , "Nickel Cadmium Battery Systems for Aerospace Support Equipment," Aerospace, IEEE Transactions on , vol.1, no.2, pp.131-139, Aug. 1963

33. Kulin, T.M.; , "Life cycle testing of a sealed 24-V, 42-Ah nickel-cadmium aircraft battery," Aerospace and Electronic Systems Magazine, IEEE, vol.12, no.10, pp.17-22, Oct 1997

34.SAFT America INC., "Nickel Cadmium aircraft battery," published online at www.saftbatteries.com/SAFT/UploadedFiles/Aircraft/PDF/4078-3.pdf

35. The Mathworks, "Battery," published online at http://www.mathworks.com/help/toolbox/physmod/powersys/ref/battery.html, assessed 19/5/11

Page 11 of 12 
36. Wu, T., Bozhko, S., Asher, G., Wheeler, P. and Thomas, D., "Fast Reduced Functional Models of Electromechanical Actuators for More-Electric Aircraft Power System Study," in SAE Power Systems Conference, November 2008, paper no. 2008-01-2859

37. Todd, R., Forsyth, A.J. , "DC-bus power quality for UAV systems during generator fault conditions," Power Electronics, Machines and Drives (PEMD 2010), 5th IET International Conference on , vol., no., pp.1-6, 19-21 April 2010

\section{CONTACT INFORMATION}

Steven Fletcher, Research Assistant, University of Strathclyde, UK

steven.fletcher@eee.strath.ac.uk

\section{ACKNOWLEDGMENTS}

This work has been carried out as part of the Rolls-Royce UTC programme 